\title{
Intra-Articular Injection of Amniotic Membrane and Umbilical Cord Particulate for the Management of Moderate to Severe Knee Osteoarthritis
}

This article was published in the following Dove Press journal: Orthopedic Research and Reviews

\author{
Olivia G Mead (D) \\ Leon P Mead \\ Leon P Mead MD PA, Naples, FL, USA
}

Correspondence: Leon P Mead

Leon Mead MD PA, 730 Goodlette Road

North, Suite 20I, Naples, FL 34102, USA

$\mathrm{Tel}+\mid$ 239-262-III

$\mathrm{Fax}+\mid$ 239-204-3104

Email orthomead@aol.com
Objective: To evaluate the long-term benefit of a single injection of amniotic membrane/ umbilical cord (AM/UC) particulate in patients with moderate to severe knee osteoarthritis (OA).

Methods: A single-center, investigator-initiated, retrospective study of patients who received intra-articular injection of $100 \mathrm{mg}$ lyophilized and micronized AM/UC for radiographically confirmed, Kellgren-Lawrence (KL) grade 3 or 4 symptomatic knee OA. Data regarding demographics, OA severity, comorbidities, treatment regimens, complications, and patient-reported outcomes were collected and assessed. Patient Global Impression of Change (PGIC) was assessed on a 7-point scale, and Global Perceived Improvement (GPI), expressed as percent improvement relative to baseline, was used to further quantify the degree of symptomatic change. Clinically important response to treatment was assessed at 12 months using simplified OMERACT-OARSI responder criteria.

Results: A total of 42 patients with KL grade 3 (36\%) and 4 (64\%) knee OA were included for analysis. Prior to injection, patient-rated pain was $6.6 \pm 1.5$ out of 10 (median: 7, range: 3-10) despite prior treatment with oral/topical NSAIDs (62\%) and intra-articular injection(s) of corticosteroids (57\%) and/or hyaluronic acid (48\%). Twelve months following AM/UC injection, 31 patients (74\%) reported significant clinical improvement via PGIC, and the OMERACT-OARSI treatment response rate was $81 \%$ (34/42). GPI of pain and function was $62 \pm 24 \%, 69 \pm 27 \%, 69 \pm 27 \%$, and $64 \pm 31 \%$ at 1, 3, 6, and 12 months, respectively. Symptomatic improvement was maintained for an average duration of $12.1 \pm 4.5$ months (median: 12, range: 3-22). One patient developed swelling in the knee within 36 hours of injection. No other adverse events or complications were reported.

Conclusion: Intra-articular injection of AM/UC particulate may be effective in alleviating pain and improving function in patients with moderate to severe knee OA, with the potential to delay total knee replacement for up to 12 months.

Keywords: amniotic membrane, injection, knee, osteoarthritis, umbilical cord

\section{Introduction}

Knee osteoarthritis (OA) is a degenerative joint disease affecting nearly 14 million individuals in the United States alone. ${ }^{1}$ Pathologic features include articular cartilage degradation, remodeling of subchondral bone, osteophyte formation, and synovial inflammation. Initially believed to be the result of wear and tear on the joints, the pathogenesis of $\mathrm{OA}$ is now known to be largely driven by a complex interplay of inflammatory mediators. ${ }^{2}$ This inflammation, coupled with progressive articular erosion, causes pain, progressive loss of function, and diminished quality of life. ${ }^{3,4}$ Given 
its high prevalence, knee OA is a leading cause of physical disability in the aging population. ${ }^{5}$

There are currently few effective conservative treatment modalities for symptomatic knee OA, none of which prevent disease progression. Analgesics, such as nonsteroidal anti-inflammatory drugs (NSAIDs), acetaminophen, and opiates, provide only modest, temporary pain relief that is often accompanied by adverse side effects, especially in the elderly knee OA population. ${ }^{6,7}$ Intraarticular injection of corticosteroids is a common treatment that targets inflammation; however, benefits may be short term, ${ }^{8,9}$ and recent findings suggest that these injections are associated with progressive articular cartilage loss and may ultimately worsen the joint's arthritic state. ${ }^{10-12}$ Furthermore, intra-articular hyaluronic acid (HA) injections have been widely used to improve joint lubrication, yet the clinical practice guidelines by the American Academy of Orthopedic Surgeons (AAOS) strongly recommend against their use, ${ }^{13}$ as they have demonstrated little to no clinical benefit. ${ }^{14}$

With the new paradigm of $\mathrm{OA}$ as an inflammationdriven disease, there has been a surge of interest in biologic therapies to alleviate pain and slow disease progression. Placenta-derived products, including cryopreserved amniotic membrane (AM) and umbilical cord (UC), are currently being investigated given their commercial availability, long history of safe use, and their known antiinflammatory, anti-scarring, and pro-regenerative properties. ${ }^{15-18}$ In a preclinical study, Raines et $\mathrm{al}^{19}$ found that intra-articular injection of $\mathrm{AM} / \mathrm{UC}$ particulate dose-dependently attenuates cartilage degeneration in a rat OA model. A recent prospective pilot study further demonstrated that one or two injections of $50 \mathrm{mg} \mathrm{AM} /$ UC particulate significantly improved pain and physical function in mild knee OA for up to 6 months. ${ }^{20}$ Given these encouraging findings, we conducted a retrospective study to assess the clinical benefit of a single intraarticular injection of $100 \mathrm{mg} \mathrm{AM} / \mathrm{UC}$ particulate in alleviating pain and promoting functional recovery in patients with moderate-to-severe knee OA.

\section{Patients and Methods}

Following the IRB review exemption and waiver of authorization by Sterling Institutional Review Board, we reviewed the medical records of consecutive patients who received an intra-articular injection of AM/UC particulate for knee OA pain by a single orthopedic surgeon in a private practice setting between May 2017 (initial use of treatment) and March 2019 (enabling 1 year of followup data at the time of data extraction). In accordance with the Declaration of Helsinki, minimum necessary data were extracted from electronic medical records, which included age, gender, body mass index (BMI), potentially limiting comorbidities, duration of symptomatic knee pain, knee OA severity, degree of joint space narrowing, baseline pain intensity by the Numeric Rating Scale (NRS), prior or concomitant therapies, patient-reported outcomes, and complications or adverse events following AM/UC treatment. Access of all patient data complied with relevant data protection and privacy regulations.

\section{Patients}

Patients were included in this study if they received a single, in-office injection of $100 \mathrm{mg} \mathrm{AM} / \mathrm{UC}$ particulate (Clarix FLO $^{\circledR}$; Tissuetech, Inc., Miami, FL, USA) for knee pain secondary to $\mathrm{OA}$ and had a clinical diagnosis of moderate to severe knee OA, which was defined as a Kellgren-Lawrence (KL) ${ }^{21}$ grade 3 or 4 based on radiographic evidence (standing anteroposterior, lateral, Rosenberg, and sunrise views). Patients were excluded if they received an intra-articular injection of corticosteroid, HA, or biologic in the target knee within 3 months of AM/ UC injection $(\mathrm{n}=14)$, had concomitant pathologies such as meniscal tear or ligament injury $(\mathrm{n}=19)$, had rheumatoid arthritis ${ }^{22}(\mathrm{n}=2)$, did not have sufficient or quantifiable follow-up data $(\mathrm{n}=31)$, or received a second injection of $\mathrm{AM} / \mathrm{UC}$ within 12 months of initial injection $(\mathrm{n}=14)$. The patient selection process is summarized in Figure 1.

\section{Intra-Articular Injection of AM/UC}

The AM/UC particulate (Clarix FLO ${ }^{\circledR}$, Amniox Medical, Miami, FL, USA) is derived from donated human placenta tissue following healthy, live, caesarian section, full-term births. Under aseptic conditions, the AM and UC are removed from the placenta and cleaned of blood. This is followed by lyophilization, micronization, and terminal sterilization. All living cells are devitalized during this processing, while the tissues' natural biological characteristics, including its anti-inflammatory properties, are preserved. ${ }^{23}$ The final AM/UC product is in a particulate (powder) form and is stored at room temperature.

Using a 21-gauge needle and a pre-filled, $12-\mathrm{mL}$ syringe with $10 \mathrm{~mL}$ fill volume of $0.9 \%$ sodium chloride (Aquastat Saline Flush Syringe, Aquabiliti ${ }^{\mathrm{TM}}$, Nashville, TN, USA), $2 \mathrm{~mL}$ saline was added to a sterile vial 


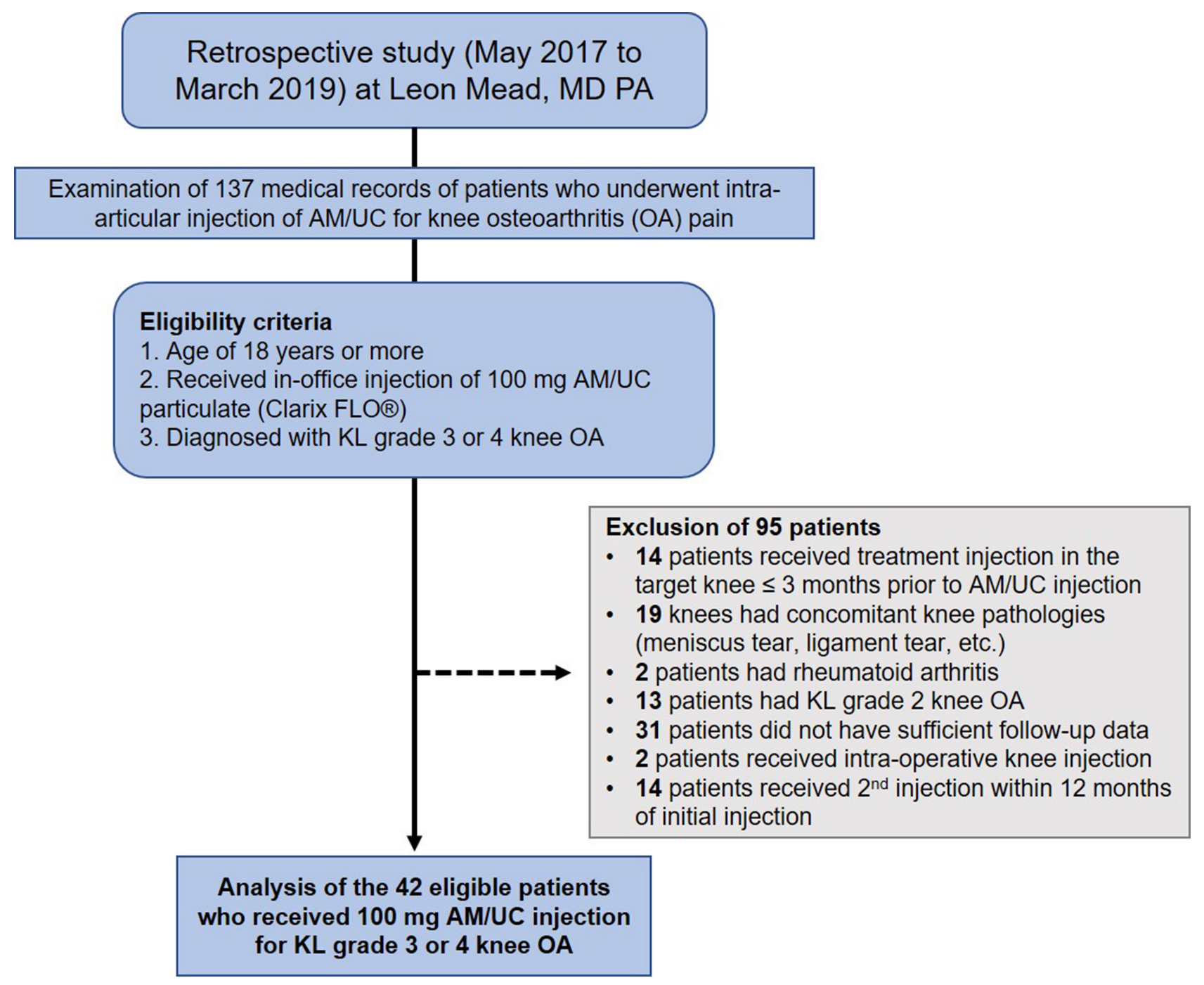

Figure I Flowchart of patient selection.

containing $100 \mathrm{mg}$ of AM/UC particulate. The AM/UC solution was left to hydrate for 45 minutes. The knee was then prepped with alcohol and 10\% povidone-iodine solution (Betadine ${ }^{\circledR}$, Purdue Frederick Co, Stamford, CT, USA), and any persistent synovial fluid was aspirated using an 18-gauge needle through a superolateral approach. Excess saline was disposed of from the prefilled syringe to leave only $3 \mathrm{~mL}$ saline remaining, and then $1 \mathrm{~mL}$ of $1 \%$ lidocaine was drawn into the syringe. The AM/UC solution was drawn from the vial with a 21gauge needle into the syringe, and the $6-\mathrm{mL}$ solution was injected into the intra-articular space via a lateral arthroscopic portal approach.

Following the injection, patients were told that they might have varying amounts of discomfort for 72 hours and could take analgesics as necessary, including NSAIDs.
Patients were allowed to resume daily activities without any restrictions. Routine follow-up visits were generally scheduled at 1 month ( \pm 2 weeks), 3 months ( \pm 1 month), 6 months ( \pm 1 month), and 12 months ( \pm 1 month) following injection. Patients returned after 12 months as needed, and their data were recorded. Given the high volume of seasonal residents, patients who had returned to their primary out-of-state residence followed up with the physician via telephone.

\section{Outcome Measures}

Patient Global Impression of Change (PGIC) was used to assess the patient's overall perceived improvement of knee pain and function compared to baseline on a 7-point Likert scale. Outcomes were graded as (1) very much improved -nearly all better; good level of functioning; minimal 
symptoms; represents a very substantial change (2) much improved - notably better with a significant reduction of symptoms; increase in the level of functioning but some symptoms remain (3) minimally improved - slightly better with little or no clinically meaningful reduction of symptoms (4) no change-symptoms remain essentially unchanged (5) minimally worse - slightly worse but may not be clinically meaningful (6) much worse-clinically significant increase in symptoms and diminished functioning or (7) very much worse - severe exacerbation of symptoms and loss of functioning. Clinically important improvement via PGIC was defined as a response of "much improved" or "very much improved". Global perceived improvement (GPI), expressed as percent improvement following treatment relative to baseline, was used to further quantify the degree of symptomatic change in pain and function at follow-up, as it has been shown to strongly correlate with percent improvement in NRS pain. ${ }^{24}$ Furthermore, lasting benefit of injection was defined as the duration of time between treatment injection and the noticeable return of knee OA symptoms.

Clinically important response to treatment was assessed using the Outcome Measures in Rheumatology (OMERACT)-Osteoarthritis Research Society International (OARSI) responder criteria, which presents the changes in three symptomatic domains (pain, function, and patient's global assessment) after treatment as a single variable. ${ }^{25}$ For the purpose of this study, we used the criteria from scenario $\mathrm{F}^{25}$ which differs from the established responder criteria (scenario D) in that it defines improvement using only relative change rather than both absolute and relative change; nevertheless, when evaluating intra-articular specific knee OA drugs, these two scenarios demonstrate nearly the same performance with only a $2 \%$ difference between active treatment effects. ${ }^{25}$ Thus, a responder was defined as having either (1) $\geq 50 \%$ improvement in pain/function, or (2) $\geq 20 \%$ improvement in pain/function and patient's global assessment $\geq 20 \%$ (PGIC score of "minimally improved" or better). Adverse events and complications were also assessed for safety and tolerability.

\section{Statistical Analysis}

All statistical analyses were carried out using IBM SPSS version 20.0. Categorical variables were described using percentages and frequencies, while continuous variables were described as mean \pm standard deviation or median (range). The Mann-Whitney U-Test and Fisher's Exact
Test were used to assess differences in outcomes between KL grades. A $P$ value $<0.05$ was considered statistically significant. Because this was a retrospective study and follow-ups were not consistent, missing data were addressed using the multiple imputation (MI) method. MI is considered the gold standard technique for missing data and is superior to listwise deletion and other traditional methods that may produce biased estimates (ie, single imputation methods). ${ }^{26,27}$ Overall, $3.3 \%$ of values were missing, with 9/33 (27\%) variables missing at least one value. The percentage of missing values ranged from $0 \%$ for demographic variables to as high as $29 \%$ for GPI at 1 month; thus, only $71 \%$ of the 43 patients would have been available for analysis under the traditional listwise deletion method. Data are primarily missing due to inconsistent or missed follow-up appointments over the 12-month period. After analyzing patterns of missing values for monotonicity, we included all analysis variables under the assumption that data were missing in a random manner. GPI (1, 3, 6, and 12 months), PGIC (1 and 6 months), baseline pain, and symptom duration were included in the imputation process. IBM SPSS Statistics version 20's "automatic" command was used, and MI by fully conditional specification was chosen. As suggested by White et al, ${ }^{28} 50 \mathrm{impu}$ tations were chosen as $45 \%$ (19/42) of cases were missing at least one value. Analyses run on each dataset were pooled according to "Rubin's rules". ${ }^{29}$ Imputed values compared reasonably well to observed values, and results using listwise deletion are similar to MI; thus, imputed results were utilized.

\section{Results}

A total of 42 patients (18 females, 24 males) met the eligibility criteria and were included for analysis (Figure 1). Patients had a mean age of $74.1 \pm 9.0$ years (range: $52-94$ ) and a mean BMI of $27.7 \pm 4.1 \mathrm{~kg} / \mathrm{m}^{2}$ (range: 20.3-35.3). Common comorbidities included hypertension $(\mathrm{n}=24$, $57 \%)$, hypercholesterolemia $(n=17,41 \%)$, heart disease $(\mathrm{n}=12,29 \%)$, thyroid disease $(\mathrm{n}=9,21 \%)$, diabetes $(\mathrm{n}=$ $8,19 \%)$, and renal disease $(\mathrm{n}=2,5 \%)$. Knee OA severity was radiographically diagnosed as $\mathrm{KL}$ grade $3(\mathrm{n}=15,36 \%)$ and $\mathrm{KL}$ grade $4(\mathrm{n}=27,64 \%)$, and joint space demonstrated narrowing by $86 \pm 19 \%$ (range: $40-100 \%$ ). Knee OA predominantly affected the medial compartment $(n=25,60 \%)$, followed by the lateral $(n=12,29 \%)$ and the patellofemoral compartment $(n=5,12 \%)$. Symptomatic pain persisted for a median duration of 4 months (range: $0.5-120$ ) despite prior treatment with oral or topical NSAIDs $(n=26,62 \%)$ and 
intra-articular injection of corticosteroid $(n=24,57 \%)$, HA $(n=20,48 \%)$, and/or platelet-rich plasma $(n=6,14 \%)$. Five patients $(12 \%)$ had no former treatment but proceeded with AM/UC injection after discussing available treatment options due to underlying conditions, personal preference, contraindications for other injection therapies, or lifestyle circumstances.

Prior to injection, patients rated pain as $6.6 \pm 1.5$ out of 10 (median: 7, range: 3-10). Twelve months following AM/UC injection, 74\% (31/42) of patients reported clinically important improvement of knee pain and function according to PGIC (Table 1). Pain and function improved by $62 \pm 24 \%, 69 \pm 27 \%, 69 \pm 27 \%$, and $64 \pm 31 \%$ at 1,3 , 6 , and 12 months, respectively, according to GPI (Table 1). Such symptomatic improvement was maintained for a mean duration of $12.1 \pm 4.5$ months (median: 12, range: $3-22)$. At 12 months, the treatment response was $81 \%(34 / 42)$ as determined by the simplified OMERACTOARSI criteria.

Of those with KL grade 3 knee OA, clinically important improvement of knee pain and function via PGIC was reported in $87 \%(13 / 15)$ of patients at 12 months. Injection of AM/UC improved pain and function for an average of $13.4 \pm 3.6$ months (range: 8-22), and the OMERACTOARSI treatment response rate was 93\% (14/15) at 12 months. In KL grade 4 patients, clinically important improvement of knee pain and function was reported in $67 \%(18 / 27)$ of patients via PGIC. Pain and function improved for an average duration of $11.5 \pm 4.9$ months (range: 3-20), and the OMERACT-OARSI treatment response rate was $74 \%(20 / 27)$ at 12 months. PGIC and
GPI are presented by KL grade in Figures 1 and 2, respectively. PGIC significantly differed between KL grade 3 and 4 patients at 3,6 , and 12 months $(P=0.028,0.025$, and 0.027 , respectively; Figure 2). Furthermore, GPI significantly differed between KL grade 3 and 4 patients at 1 month $(P=0.026$; Figure 3$)$. No other outcomes significantly differed by KL grade throughout the study.

After injection, one patient developed swelling in the knee within 36 hours, after which time, the patient reported progressive improvement of the knee to the point where he was largely pain free at 6 weeks with an increased range of motion. This patient had underlying heart disease, diabetes, hypertension, renal disease, and thyroid disease and had undergone knee arthroplasty on the same knee by a different surgeon 8 years prior. No other adverse events or complications were reported.

During the 12-month follow-up period, one patient received a single injection of cross-linked HA (Gel-One ${ }^{\circledR}$, Zimmer Biomet Holdings, Inc, Warsaw, IN, USA) 8 months after AM/UC injection, as the patient began to have discomfort when climbing stairs. This patient was lost to follow up afterwards. There was only one patient that underwent planned total knee arthroplasty (TKA) 9 months after $\mathrm{AM} / \mathrm{UC}$ injection. No other patients received TKA during the 12-month follow-up period.

\section{Discussion}

Current conservative measures for knee OA consist of weight management, activity modification with nonimpact aerobic exercise, physical therapy, NSAIDs, HA injections, and corticosteroid injection. However, there is

Table I Patient-Reported Outcomes Following Intra-Articular Injection of Amniotic Membrane/Umbilical Cord Particulate

\begin{tabular}{|c|c|c|c|c|}
\hline & Month I & Month 3 & Month 6 & Month 12 \\
\hline \multicolumn{5}{|l|}{ KL Grade 3-4 $(N=42)$} \\
\hline GPI (\%) & $\begin{array}{l}62 \pm 24 \\
60(0-100)\end{array}$ & $\begin{array}{l}69 \pm 27 \\
75(0-100)\end{array}$ & $\begin{array}{l}69 \pm 27 \\
77.5(0-100)\end{array}$ & $\begin{array}{l}64 \pm 31 \\
75(0-100)\end{array}$ \\
\hline \multicolumn{5}{|l|}{ PGIC } \\
\hline Very much improved & $19(45 \%)$ & $25(60 \%)$ & $24(57 \%)$ & $24(57 \%)$ \\
\hline Much improved & $14(33 \%)$ & II (26\%) & $10(24 \%)$ & $7(17 \%)$ \\
\hline Minimally improved & $7(17 \%)$ & $3(7 \%)$ & $5(12 \%)$ & $4(9 \%)$ \\
\hline No Change & $2(5 \%)$ & $3(7 \%)$ & $3(7 \%)$ & $7(17 \%)$ \\
\hline Minimally Worse & $0(0 \%)$ & $0(\%)$ & $0(0 \%)$ & $0(0 \%)$ \\
\hline Much Worse & $0(0 \%)$ & $0(\%)$ & $0(0 \%)$ & $0(0 \%)$ \\
\hline Very Much Worse & $0(0 \%)$ & $0(\%)$ & $0(0 \%)$ & $0(0 \%)$ \\
\hline Cases Imputed (N) & 12 & 3 & 1 & 3 \\
\hline
\end{tabular}

Abbreviations: GPI, Global Perceived Improvement; KL, Kellgren-Lawrence; PGIC, Patient Global Impression of Change. 


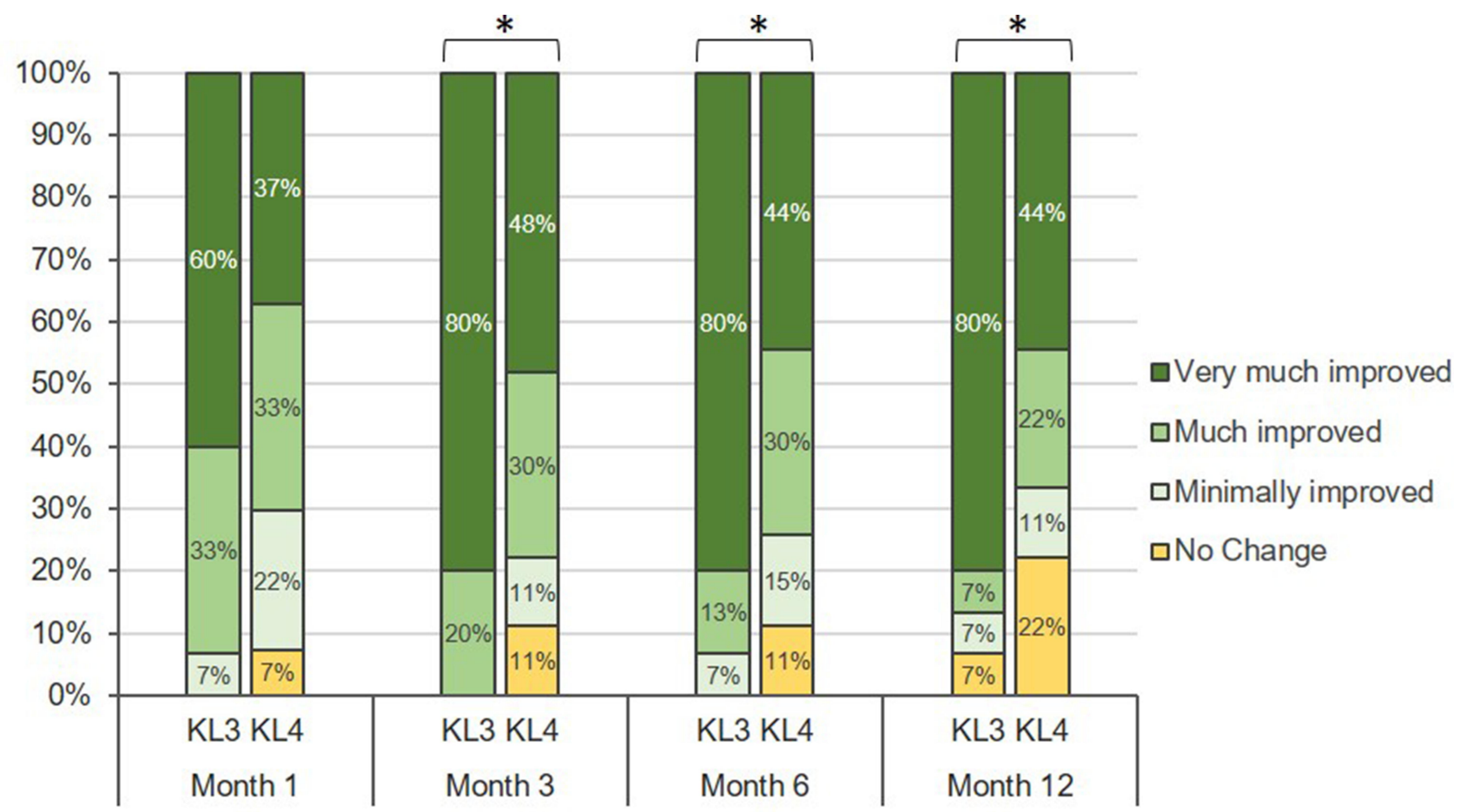

Figure 2 PGIC by KL Grade. PGIC indicates Patient Global Impression of Change on a 7-point Likert Scale. Patients with Kellgren-Lawrence (KL) grade 3 knee osteoarthritis (OA) reported greater improvement at 3,6, and 12 months compared with those with KL grade 4 knee OA (* denotes $P<0.05$ ).

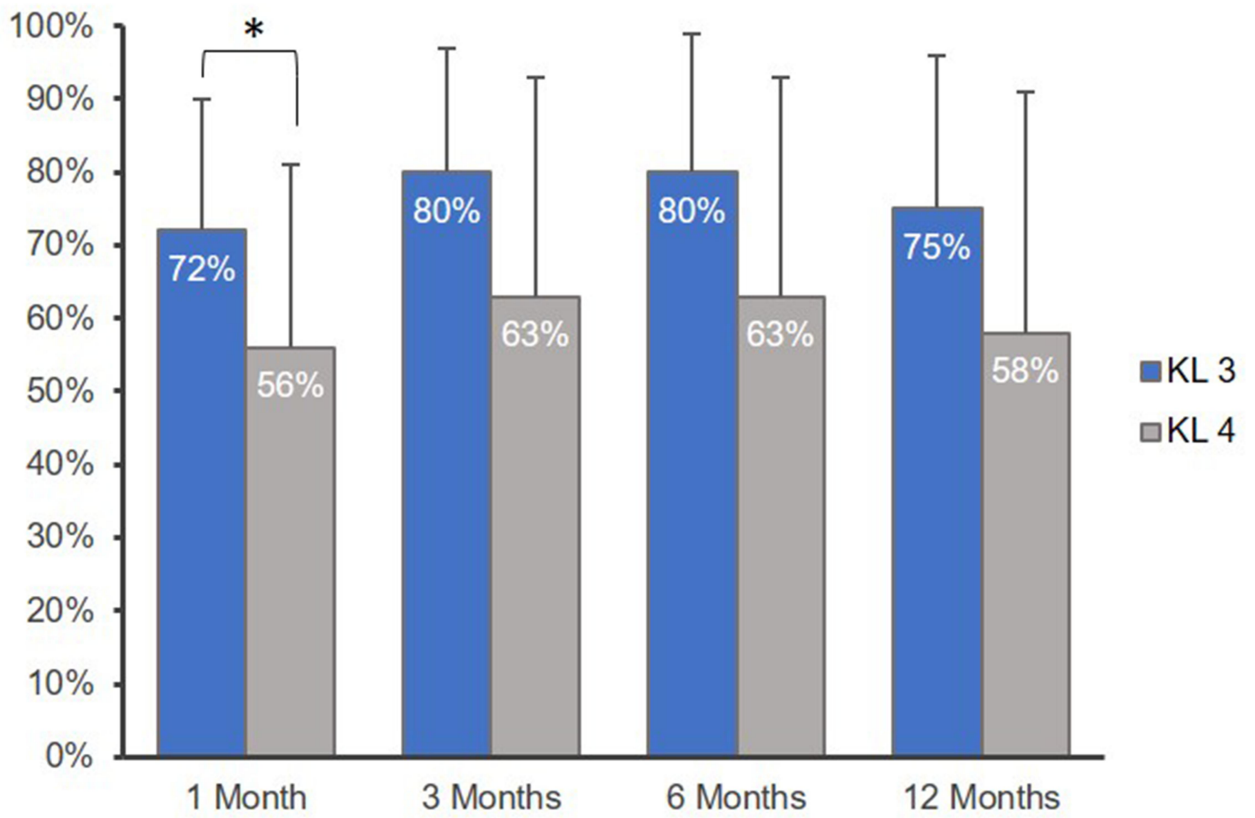

Figure 3 GPI by KL Grade. GPI indicates Global Perceived Improvement (scale range, 0-100\%). Patients with Kellgren-Lawrence (KL) grade 3 knee osteoarthritis (OA) reported significantly greater improvement at I month compared to those with $\mathrm{KL}$ grade 4 knee $\mathrm{OA}(*$ denotes $P<0.05)$. Error bars indicate standard deviation.

inconsistency among knee OA treatment guideline recommendations, particularly regarding HA and corticosteroid injections. The AAOS 2013 guidelines strongly recommend against the use of $\mathrm{HA},{ }^{13}$ while the American College of Rheumatology (ACR) guidelines recommend against its use unless other conservative measures have been exhausted; ${ }^{30}$ conversely, OARSI recently altered their 2014 recommendation of HA injections from uncertain to conditionally recommended in $2019 .{ }^{31}$ Furthermore, both ACR and OARSI recommend the use 
of corticosteroid, ${ }^{30,31}$ while the AAOS's recommendation is inconclusive. ${ }^{13}$ In the present study, patients reported moderate-to-severe knee pain at baseline (median: 7) despite prior intra-articular injection of corticosteroid $(57 \%)$ or HA $(48 \%)$. This is presumably due to the advanced radiographic severity of our study sample, which comprised only those with KL grade 3 and 4 OA. In fact, several studies have demonstrated that patients with KL 3 and 4 knee OA are less likely to respond to an injection of corticosteroid ${ }^{32-34}$ or $\mathrm{HA}^{35-37}$ when compared to those with KL 1 or 2 knee OA. A recent metaanalysis found that injection of HA is only effective in reducing pain in KL 1-3 knee OA patients, as this effect was no longer observed when including studies with KL 4 knee OA. ${ }^{38}$ The observed decrease in efficacy upon including studies with KL 4 knee OA was statistically significant, further supporting the notion that patients with end-stage knee $\mathrm{OA}$ are less likely to respond to conservative treatment measures. ${ }^{38}$

When non-operative treatment fails and quality of life becomes notably compromised, surgical intervention is often considered. ${ }^{39}$ Surgical treatments for knee OA include osteotomy, arthroscopy, cartilage repair, and partial or total knee arthroplasty (TKA). In fact, more than $50 \%$ of knee OA patients in the United States will eventually undergo TKA, with the majority of cases having KL grade 3 or $4 \mathrm{OA}^{40}$ Yet, knee OA patients spend nearly half of their post-diagnosis life ( $13 \pm 10$ years) waiting for TKA after exhausting available non-surgical options, ${ }^{41}$ underscoring the need for new conservative therapies that can effectively manage advanced symptomatic knee OA. In the present study, $74 \%$ of patients rated knee pain and function as "much improved" or "very much improved" 12 months after AM/UC injection, which is in accordance with the 12-month treatment response rate of $81 \%$ according to the simplified OMERACT-OARSI criteria. This study also disclosed that intra-articular injection of AM/ UC particulate is generally safe as there were no reported complications or adverse events aside from one case of knee swelling within 36 hours of injection. Lastly, symptomatic pain and function improved for an average duration of 12 months, with one patient delaying TKA for 9 months following $\mathrm{AM} / \mathrm{UC}$ injection. Collectively, these results suggest that intra-articular injection of $\mathrm{AM} / \mathrm{UC}$ may be an effective treatment option for patients with moderate-to-severe knee $\mathrm{OA}$, with the potential to delay TKA.
The aforementioned clinical benefit of AM/UC particulate can be attributed to its anti-inflammatory properties. The underlying mechanism may be due in part to AM/ UC's ability to downregulate IL- $1 \beta,{ }^{16,42}$ which has long been considered a central mediator of cartilage loss in OA by upregulating the major extracellular proteolytic enzymes in cartilage degeneration. ${ }^{43}$ Suppression of IL$1 ß$ pathways has shown to be effective in reducing pain and preserving articular cartilage in animal models as well as humans. ${ }^{44,45}$ The therapeutic effects of AM/UC may also be due to its ability to suppress TGF- $\beta$ signaling, ${ }^{46,47}$ which is highly associated with inflammatory states. This notion is derived from in vitro and in vivo studies that have found TGF- $\beta$ signaling to play a pathogenic role in the development of knee $\mathrm{OA}^{48}$ Animal studies have shown that suppression of TGF- $\beta$ signaling in the subchondral bone attenuates pathological OA changes including articular cartilage degeneration. ${ }^{49,50}$ In addition, AM/ UC's innate, major biochemical component, ie, HC-HA /PTX3, has been shown to be responsible in part for its anti-inflammatory, anti-scarring, and pro-regenerative effects. ${ }^{51}$ Unlike high molecular weight $\mathrm{HA},{ }^{52}$ which is conventionally used for the treatment of knee OA, HC-HA /PTX3 has been shown to significantly promote apoptosis of activated neutrophils ${ }^{53,54}$ and polarization of M2 macrophages. ${ }^{54}$ Collectively, these actions may help to resolve inflammation and attenuate cartilage degradation, both of which play an important role in the pathology and clinical manifestations of OA. ${ }^{2,3}$

The present study has several limitations. First and foremost, there was no control group due to the retrospective nature of this study. As a result, we had no means of determining the placebo effect and thus the overall treatment effect. However, the efficacy of conservative knee $\mathrm{OA}$ treatments are well documented in the literature and serve as a historical control. Furthermore, patients did not have follow-up data at every time point, as many patients cancel follow-up appointments when symptoms are well controlled. As a result, the MI technique was implemented to account for missing data, which may have affected data accuracy. However, the listwise analysis revealed that the results changed to a very minimal degree. Lastly, GPI was used in place of the NRS pain scale; it is a simple way to gage improvement, is commonly used in this clinical practice, and thus was better documented in the patients' medical records. The perception of improvement is advantageous as it can be considered an integrated response that includes not only the patient's perceived pain 
relief but also improvements in functional state and quality of life. ${ }^{55}$ Numerous studies have found a moderate overall agreement between patient-reported percentage pain reduction and calculated percentage pain reduction from NRS pain scores, suggesting that these indices can be used interchangeably. ${ }^{56-58}$ However, it should be noted that there is a discrepancy in the degree of improvement between these two measures, with overestimations of selfreported percentages ranging from $2.6 \%{ }^{57}$ to $16 \%{ }^{58}$ In the current study, however, post hoc analysis demonstrated that the average discrepancy between self-reported and calculated percent improvement was $2.6 \pm 8.8 \%$ (median: $0 \%$, range: -10 to $+25 \%$ ) from 50 NRS follow-up values, suggesting that overreporting improvement is unlikely to significantly affect the validity of these results. Despite these limitations, this retrospective study provides insightful, real-world evidence from an orthopedic clinical practice that suggest $\mathrm{AM} / \mathrm{UC}$ particulate injection may be beneficial in patients with advanced knee OA who have exhausted other conservative treatment options. Prospective randomized controlled studies are warranted to validate the efficacy, safety, and long-term benefit of $\mathrm{AM} / \mathrm{UC}$ in treating symptomatic knee OA.

\section{Conclusion}

In conclusion, intra-articular injection of $\mathrm{AM} / \mathrm{UC}$ particulate may be a safe and effective treatment option in patients with moderate to severe knee OA with the potential to delay TKA. Prospective randomized controlled studies are necessary to validate its safety, efficacy, and longterm benefit.

\section{Disclosure}

Olivia G Mead reports personal fees from TissueTech, Inc., outside the submitted work. The authors report no other conflicts of interest in this work.

\section{References}

1. Lawrence RC, Felson DT, Helmick CG, et al. Estimates of the prevalence of arthritis and other rheumatic conditions in the United States. Part II. Arthritis Rheum. 2008;58(1):26-35. doi:10.1002/ art.23176

2. Goldring MB, Otero M. Inflammation in osteoarthritis. Curr Opin Rheumatol. 2011;23(5):471-478. doi:10.1097/BOR.0b013e328349c $2 \mathrm{~b} 1$

3. Li L, Li Z, Li Y, Hu X, Zhang Y, Fan P. Profiling of inflammatory mediators in the synovial fluid related to pain in knee osteoarthritis. BMC Musculoskelet Disord. 2020;21(1):99. doi:10.1186/s12891-0203120-0

4. Nuki G. Osteoarthritis: a problem of joint failure. Z Rheumatol. 1999;58(3):142-147.
5. Guccione AA, Felson DT, Anderson JJ, et al. The effects of specific medical conditions on the functional limitations of elders in the Framingham Study. Am J Public Health. 1994;84(3):351-358. doi:10.2105/AJPH.84.3.351

6. da Costa BR, Nuesch E, Kasteler R, et al. Oral or transdermal opioids for osteoarthritis of the knee or hip. Cochrane Database Syst Re. 2014;(9):Cd003115.

7. Osani MC, Vaysbrot EE, Zhou M, McAlindon TE, Bannuru RR. Duration of symptom relief and early trajectory of adverse events for oral nsaids in knee osteoarthritis: a systematic review and meta-analysis. Arthritis Care Res. 2019.

8. Saltychev M, Mattie R, McCormick Z, Laimi K. The magnitude and duration of the effect of intra-articular corticosteroid injections on pain severity in knee osteoarthritis - a systematic review and meta-analysis. Am J Phys Med Rehabil. 2020. doi:10.1097/ PHM.0000000000001384

9. Levy DM, Petersen KA, Scalley Vaught M, Christian DR, Cole BJ. Injections for knee osteoarthritis: corticosteroids, viscosupplementation, platelet-rich plasma, and autologous stem cells. Arthroscopy. 2018;34(5):1730-1743. doi:10.1016/j.arthro.2018.02.022

10. Kompel AJ, Roemer FW, Murakami AM, Diaz LE, Crema MD, Guermazi A. Intra-articular corticosteroid injections in the hip and knee: perhaps not as safe as we thought? Radiology. 2019;293 (3):656-663. doi:10.1148/radiol.2019190341

11. McAlindon TE, LaValley MP, Harvey WF, et al. Effect of Intra-articular triamcinolone vs saline on knee cartilage volume and pain in patients with knee osteoarthritis: a randomized clinical trial. JAMA. 2017;317(19):1967-1975. doi:10.1001/jama.2017.5283

12. Zeng C, Lane NE, Hunter DJ, et al. Intra-articular corticosteroids and the risk of knee osteoarthritis progression: results from the osteoarthritis initiative. Osteoarthr Cartilage. 2019;27(6):855-862. doi:10. 1016/j.joca.2019.01.007

13. Jevsevar DS. Treatment of osteoarthritis of the knee: evidence-based guideline, 2nd Edition. J Am Acad Orthop Surg. 2013;21(9):571-576.

14. Rutjes AW, Juni P, da Costa BR, Trelle S, Nuesch E, Reichenbach S. Viscosupplementation for osteoarthritis of the knee: a systematic review and meta-analysis. Ann Intern Med. 2012;157(3):180-191. doi:10.7326/0003-4819-157-3-201208070-00473

15. Liu J, Sheha H, Fu Y, Liang L, Tseng SC. Update on amniotic membrane transplantation. Expert Rev Ophthalmol. 2010;5 (5):645-661. doi:10.1586/eop.10.63

16. Tseng SC, Espana EM, Kawakita T, et al. How does amniotic membrane work? Ocul Surf. 2004;2(3):177-187. doi:10.1016/S15420124(12)70059-9

17. Tighe S, Mead OG, Lee A, Tseng SCG. Basic science review of birth tissue uses in ophthalmology. Taiwan $J$ Ophthalmol. 2020;10 (1):3-12. doi:10.4103/tjo.tjo_4_20

18. Huddleston HP, Cohn MR, Haunschild ED, Wong SE, Farr J, Yanke AB. Amniotic product treatments: clinical and basic science evidence. Curr Rev Musculoskelet Med. 2020;13(2):148-154. doi:10.1007/s12178-020-09614-2

19. Raines AL, Shih MS, Chua L, Su CW, Tseng SC, O'Connell J. Efficacy of particulate amniotic membrane and umbilical cord tissues in attenuating cartilage destruction in an osteoarthritis model. Tissue Eng Part A. 2017;23(1-2):12-19. doi:10.1089/ten.tea.2016.0088

20. Castellanos R, Tighe S. Injectable amniotic membrane/umbilical cord particulate for knee osteoarthritis: a prospective, single-center pilot study. Pain Med (Malden, Mass). 2019. doi:10.1093/pm/pnz143

21. Kellgren JH, Lawrence JS. Radiological assessment of osteo-arthrosis. Ann Rheum Dis. 1957;16(4):494-502. doi:10.1136/ard.16.4.494

22. Karlson EW, Sanchez-Guerrero J, Wright EA, et al. A connective tissue disease screening questionnaire for population studies. Ann Epidemiol. 1995;5(4):297-302. doi:10.1016/1047-2797(94)00096-C

23. Tan E, Cooke M, Mandrycky C, et al. Structural and biological comparison of cryopreserved and fresh amniotic membrane tissues. J Biomater Tiss Eng. 2014;4. 
24. Theodore BR, Olamikan S, Keith RV, Gofeld M. Validation of self-reported pain reduction after diagnostic blockade. Pain Med (Malden, Mass). 2012;13(9):1131-1136. doi:10.1111/j.1526-4637.20 12.01442.x

25. Pham T, van der Heijde D, Altman RD, et al. Omeract-oarsi initiative: osteoarthritis research society international set of responder criteria for osteoarthritis clinical trials revisited. Osteoarthr Cartilage. 2004;12(5):389-399. doi:10.1016/j.joca.20 04.02.001

26. Jamshidian M, Mata M. 2 - Advances in analysis of mean and covariance structure when data are incomplete. In: Lee S-Y, editor. Handbook of Latent Variable and Related Models. Amsterdam: North-Holland; 2007:21-44.

27. Cox BE, McIntosh K, Reason RD, Terenzini PT. Working with missing data in higher education research: a primer and real-world example. Rev High Educ. 2014;37(3):377-402. doi:10.1353/rhe.2014.0026

28. White IR, Royston P, Wood AM. Multiple imputation using chained equations: issues and guidance for practice. Stat Med. 2011;30 (4):377-399. doi:10.1002/sim.4067

29. Rubin DB. Multiple Imputation for Nonresponse in Surveys. Vol. 81. John Wiley \& Sons; 2004.

30. Kolasinski SL, Neogi T, Hochberg MC, et al. 2019 American College of Rheumatology/arthritis foundation guideline for the management of osteoarthritis of the hand, hip, and knee. Arthrit Care Res. 2020;72 (2):149-162. doi:10.1002/acr.24131

31. Bannuru RR, Osani MC, Vaysbrot EE, et al. Oarsi guidelines for the non-surgical management of knee, hip, and polyarticular osteoarthritis. Osteoarthr Cartilage. 2019;27(11):1578-1589. doi:10. 1016/j.joca.2019.06.011

32. Matzkin EG, Curry EJ, Kong Q, Rogers MJ, Henry M, Smith EL. Efficacy and treatment response of intra-articular corticosteroid injections in patients with symptomatic knee osteoarthritis. $J \mathrm{Am}$ Acad Orthop Sur. 2017;25(10):703-714. doi:10.5435/JAAOS-D-1600541

33. Arden NK, Reading IC, Jordan KM, et al. A randomised controlled trial of tidal irrigation vs corticosteroid injection in knee osteoarthritis: the kivis study. Osteoarthr Cartilage. 2008;16(6):733-739. doi:10.1016/j.joca.2007.10.011

34. Maricar N, Parkes MJ, Callaghan MJ, et al. Structural predictors of response to intra-articular steroid injection in symptomatic knee osteoarthritis. Arthritis Res Ther. 2017;19(1):88. doi:10.1186/s13075017-1292-2

35. Altman RD, Rosen JE, Bloch DA, Hatoum HT, Korner P, DoubleBlind A. Randomized, saline-controlled study of the efficacy and safety of euflexxa for treatment of painful osteoarthritis of the knee, with an open-label safety Extension (the Flexx Trial). Semin Arthritis Rheu. 2009;39(1):1-9. doi:10.1016/j.semarthrit. 2009.04.001

36. Eymard F, Chevalier X, Conrozier T. Obesity and radiological severity are associated with viscosupplementation failure in patients with knee osteoarthritis. J Orthop Res. 2017;35(10):2269-2274. doi:10.1002/ jor.23529

37. Strand V, McIntyre LF, Beach WR, Miller LE, Block JE. Safety and efficacy of us-approved viscosupplements for knee osteoarthritis: a systematic review and meta-analysis of randomized, saline-controlled trials. J Pain Res. 2015;8:217-228.

38. Nicholls M, Shaw P, Niazi F, Bhandari M, Bedi A. The impact of excluding patients with end-stage knee disease in intra-articular hyaluronic acid trials: a systematic review and meta-analysis. Adv Ther. 2019;36(1):147-161. doi:10.1007/s12 325-018-0847-1

39. Steinhaus ME, Christ AB, Cross MB. Total knee arthroplasty for knee osteoarthritis: support for a foregone conclusion? HSS J. 2017;13(2):207-210. doi:10.1007/s11420-017-9558-4
40. Weinstein AM, Rome BN, Reichmann WM, et al. Estimating the burden of total knee replacement in the United States. $J$ Bone Joint Surg Am. 2013;95(5):385-392. doi:10.2106/JBJS.L.00206

41. Losina E, Paltiel AD, Weinstein AM, et al. Lifetime medical costs of knee osteoarthritis management in the united states: impact of extending indications for total knee arthroplasty. Arthritis Care Res (Hoboken). 2015;67(2):203-215.

42. Solomon A, Rosenblatt M, Monroy D, Ji Z, Pflugfelder SC, Tseng SC. Suppression of interleukin 1alpha and interleukin 1beta in human limbal epithelial cells cultured on the amniotic membrane stromal matrix. Brit J Ophthalmol. 2001;85(4):444-449. doi:10.1136/ bjo.85.4.444

43. Daheshia M, Yao JQ. The Interleukin 1beta pathway in the pathogenesis of osteoarthritis. $J$ Rheumatol. 2008;35(12):2306-2312. doi:10.3899/jrheum.080346

44. Chevalier X, Giraudeau B, Conrozier T, Marliere J, Kiefer P, Goupille P. Safety study of intraarticular injection of interleukin 1 receptor antagonist in patients with painful knee osteoarthritis: a multicenter study. J Rheumatol. 2005;32(7):1317-1323.

45. Frisbie DD, Ghivizzani SC, Robbins PD, Evans CH, McIlwraith CW. Treatment of experimental equine osteoarthritis by in vivo delivery of the equine interleukin-1 receptor antagonist gene. Gene Ther. 2002;9 (1):12-20. doi:10.1038/sj.gt.3301608

46. Lee SB, Li DQ, Tan DT, Meller DC, Tseng SC. Suppression of Tgf-Beta signaling in both normal conjunctival fibroblasts and pterygial body fibroblasts by amniotic membrane. Curr Eye Res. 2000;20(4):325-334. doi:10.1076/0271-3683(200004)2041-5FT325

47. Tseng SC, Li DQ, Ma X. Suppression of transforming growth factor-beta isoforms, tgf-beta receptor type ii, and myofibroblast differentiation in cultured human corneal and limbal fibroblasts by amniotic membrane matrix. J Cell Physiol. 1999;179(3):325-335. doi:10.1002/ (SICI) 1097-4652(199906)179:3<325::AID-JCP10>3.0.CO;2-X

48. Shen J, Li S, Chen D. Tgf-Beta signaling and the development of osteoarthritis. Bone Res. 2014;2.

49. Cui Z, Crane J, Xie H, et al. Halofuginone attenuates osteoarthritis by inhibition of tgf-beta activity and h-type vessel formation in subchondral bone. Ann Rheum Dis. 2016;75(9):1714-1721. doi:10.1136/ annrheumdis-2015-207923

50. Zhen G, Wen C, Jia X, et al. Inhibition of Tgf-Beta signaling in mesenchymal stem cells of subchondral bone attenuates osteoarthritis. Nat Med. 2013;19(6):704-712. doi:10.1038/nm.3143

51. Tseng SC. Hc-Ha/Ptx3 purified from amniotic membrane as novel regenerative matrix: insight into relationship between inflammation and regeneration. Invest Ophthalmol Vis Sci. 2016;57(5):ORSFh1-8. doi:10.1097/j.pain.0000000000000379

52. He H, Li W, Tseng DY, et al. Biochemical Characterization and Function of complexes formed by hyaluronan and the heavy chains of Inter-Alpha-Inhibitor $(\mathrm{Hc} * \mathrm{Ha})$ purified from extracts of human amniotic membrane. J Biol Chem. 2009;284(30):20136-20146. doi:10.1074/jbc.M109.021881

53. He H, Li W, Chen SY, et al. Suppression of activation and induction of apoptosis in Raw264.7 Cells by amniotic membrane extract. Invest Ophthalmol Vis Sci. 2008;49(10):4468-4475. doi:10.1167/iovs.08-1781

54. He H, Zhang S, Tighe S, Son J, Tseng SC. Immobilized heavy chain-hyaluronic acid polarizes lipopolysaccharide-activated macrophages toward M2 phenotype. J Biol Chem. 2013;288 (36):25792-25803. doi:10.1074/jbc.M113.479584

55. Cormier S, Lavigne GL, Choinière M, Rainville P. Expectations predict chronic pain treatment outcomes. Pain. 2016;157(2):329-338.

56. Pratici E, Nebout S, Merbai N, Filippova J, Hajage D, Keita H. An observational study of agreement between percentage pain reduction calculated from visual analog or numerical rating scales versus that reported by parturients during labor epidural analgesia. Int $J$ Obstet Anesth. 2017;30:39-43. doi:10.1016/j.ijoa.2017.02.001 
57. Cepeda MS, Africano JM, Polo R, Alcala R, Carr DB. Agreement between percentage pain reductions calculated from numeric rating scores of pain intensity and those reported by patients with acute or cancer pain. Pain. 2003;106(3):439-442. doi:10.1016/j.pain.2003. 09.006
58. Cushman D, McCormick Z, Casey E, Plastaras CT. Discrepancies in describing pain: is there agreement between numeric rating scale scores and pain reduction percentage reported by patients with musculoskeletal pain after corticosteroid injection? Pain Med (Malden, Mass). 2015;16(5):870-876. doi:10.1111/pme.12669

\section{Publish your work in this journal}

Orthopedic Research and Reviews is an international, peer-reviewed, open access journal that focusing on the patho-physiology of the musculoskeletal system, trauma, surgery and other corrective interventions to restore mobility and function. Advances in new technologies, materials, techniques and pharmacological agents are particularly

Submit your manuscript here: https://www.dovepress.com/orthopedic-research-and-reviews-journa welcome. The manuscript management system is completely online and includes a very quick and fair peer-review system, which is all easy to use. Visit http://www.dovepress.com/testimonials.php to read real quotes from published authors. 